\title{
En un mundo de lunares infinitos Exhibición de Yayoi Kusama
}

In a world of inifite dots. An exhibition by Yayoi Kusama

\author{
DOI: https://doi.org/10.5377/koot.v0i11.10731 \\ URI: http://hdl.handle.net/11298/1187
}

\author{
Alejandra Flamenco \\ Diseñadora de interiores \\ ale.flamenco29@gmail.com
}

\section{Resumen}

Fecha de aceptación: noviembre 2019

En galería David Zwirner, de la ciudad de Nueva York, tuvo lugar durante diciembre de 2019 la exhibición "Everyday I Pray for Love" (Todos los días rezo por el amor) de la escultora, pintora y escritora japonesa Yayoi Kusama. Una artista reconocida a nivel mundial por sus obras - usualmente una repetición de formas, comúnmente lunares en una diversidad de colores y composiciones-al igual que por sus cuartos de infinitos reflejados los cuales son inspirados en el universo, tema recurrente en su trabajo.

Palabras clave: Arte moderno. Escultura moderna. Pintura moderna. Estética. Crítica de arte.

\begin{abstract}
The David Zwirner Gallery in New York City exhibited "Every day I Pray for Love" last December, a work of art by internationally reknown Japanese sculptor, writer and painter Yayoi Kusama. Her work is usually a repetition of forms, generally dots - in a variety of colors and compositions. In like manner, she is also known by the infinite reflections she uses: an inspiration on the universe; a recurring theme in her works.
\end{abstract}

Key words: Modern art. Modern sculpture. Modern painting. Esthetic. Art critic. 


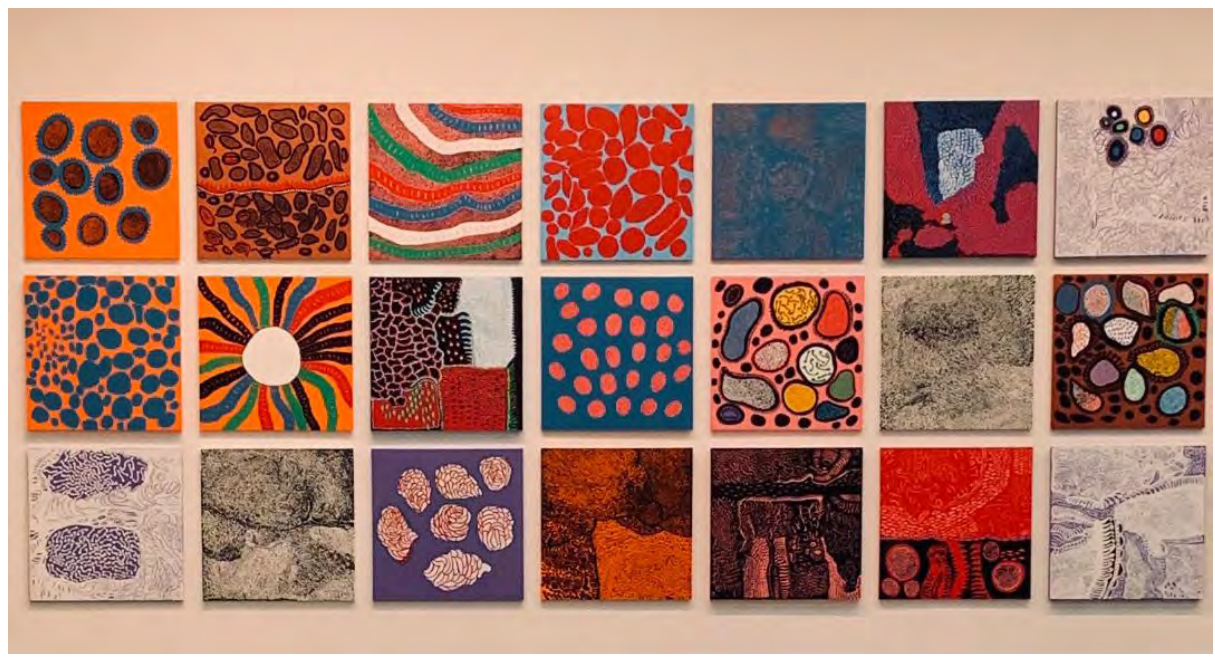

En galería David Zwirner, de la ciudad de Nueva York, tuvo lugar durante diciembre la exhibición "Everyday I Pray for Love" (Todos los días rezo por el amor) de la escultora, pintora y escritora japonesa Yayoi Kusama. Una artista reconocida a nivel mundial por sus obras - usualmente una repetición de formas, comúnmente lunares en una diversidad de colores y composiciones-al igual que por sus cuartos de infinitos reflejados los cuales son inspirados en el universo, tema recurrente en su trabajo.

Se considera que Yayoi Kusama nació en Matsumoto, Japón en 1929. Presentó sus obras por primera vez en Japón en 1952.

Durante la mitad de la década de 1960 se estableció en Nueva York como una artista de vanguardia importante escenificando acontecimientos innovadores e influyentes. Su trabajo ganó amplio reconocimiento a finales de la década de 1980 siguiendo un número de exhibiciones internacionales en solitario incluyendo presentaciones en el Center for International Contemporary Arts en Nueva York y el Museum of Modern Art en Oxford, ambas llevadas a cabo en 1989. A partir de ellas su trabajo ha sido exhibido y galardonado en diferentes ciudades en Europa, América del Norte, América del Sur, Oceanía y Asia. En 2017 un museo en honor a la artista abrió sus puertas en Tokio, ahora con su quinta exhibición titulada "Espíritus de Agregación" que termina el 31 de enero de 2020. A sus 90 años Kusama vive y trabaja desde Tokio, Japón. (Zwirner, 2019). 
Tuve la oportunidad de visitar su exhibición en Nueva York que reúne alguna de las obras más recientes de Kusama, entre pinturas y esculturas. Para comenzar, visité el más reciente Infinity Mirrored Room o 'Cuarto de infinito reflejado' titulado "Dancing Lights that Flew Up to the Universe" ("Luces danzantes que volaron hacia el universo'). Es una experiencia inmersiva en la que durante 60 segundos (tiempo escogido por la artista), luces LED suspendidas y en forma de esferas mantienen una secuencia intermitente entre encendido y apagado hasta llegar a un color rojo intenso que inunda la habitación volviendo a los espectadores parte de la obra en la que me sentí dentro de una constelación singular de estrellas en donde mi percepción de espacio cambió con los reflejos en los espejos que hacían que lo que estaba a mi alrededor se multiplicara decenas de veces hasta desintegrarse en el fondo de la habitación. Esta pieza es una muestra de la determinación de la artista a transmitir a través de sus piezas un "universo eterno ilimitado [y] la eternidad de las interrelaciones". (The Broad, 2017).

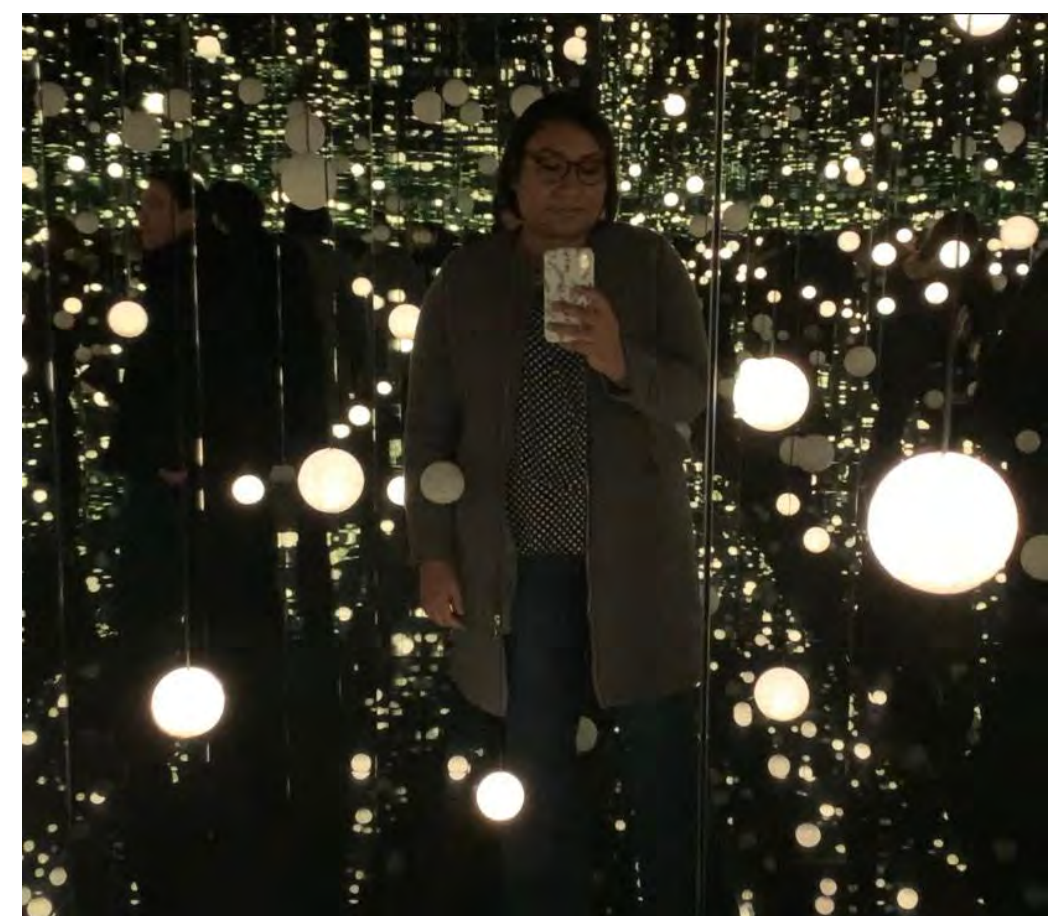




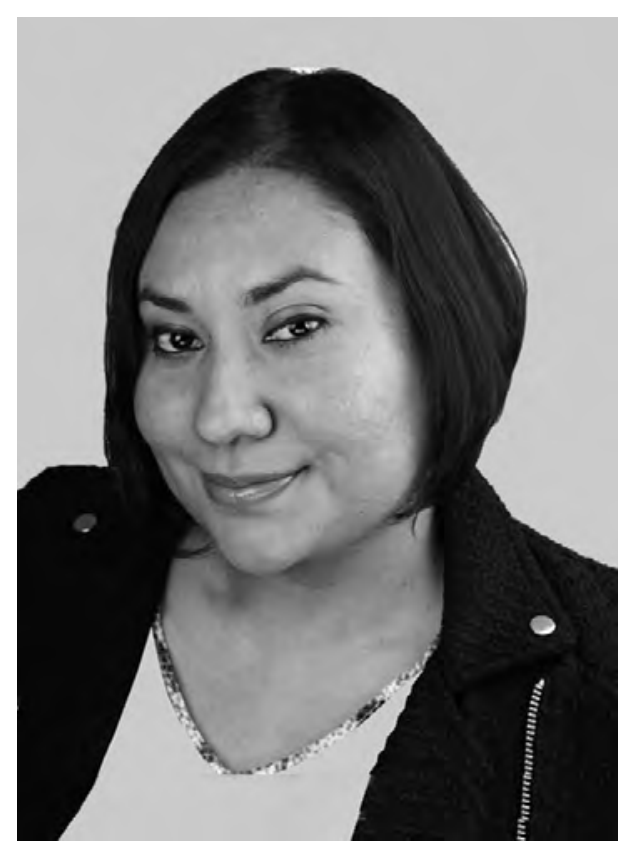

Alejandra Flamenco

En la galería adjunta se exhibieron sus más recientes pinturas, parte de la serie My Eternal Soul (Mi alma eterna), que son una exploración de líneas y formas abstractas detalladas en una variedad de colores y patrones las cuales se complementan con una instalación de piso compuesta por formas orgánicas como nubes o gotas derramadas en acero inoxidable cuyos reflejos refuerzan la idea de infinito.

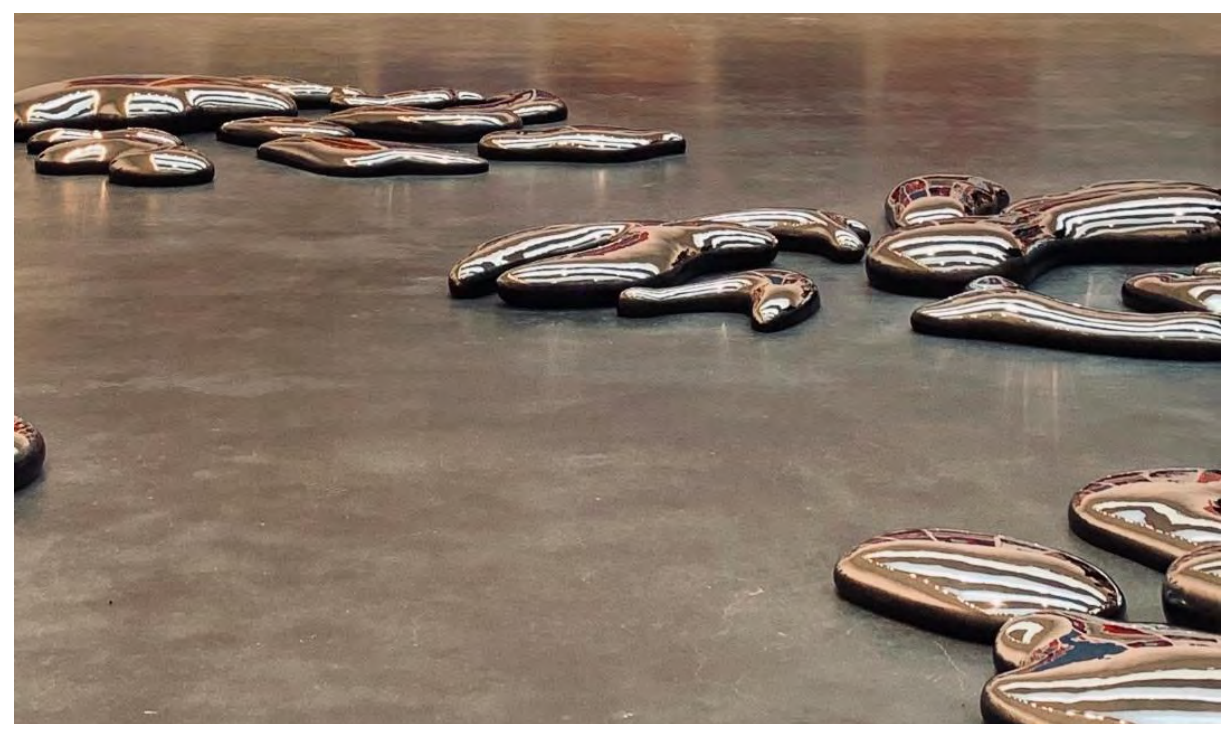


Como espectadora navegar a través de estos elementos me generó una sensación de asombro y descubrimiento al observar cómo cada forma es diferente de la otra pero que en conjunto generaban una composición homogénea.

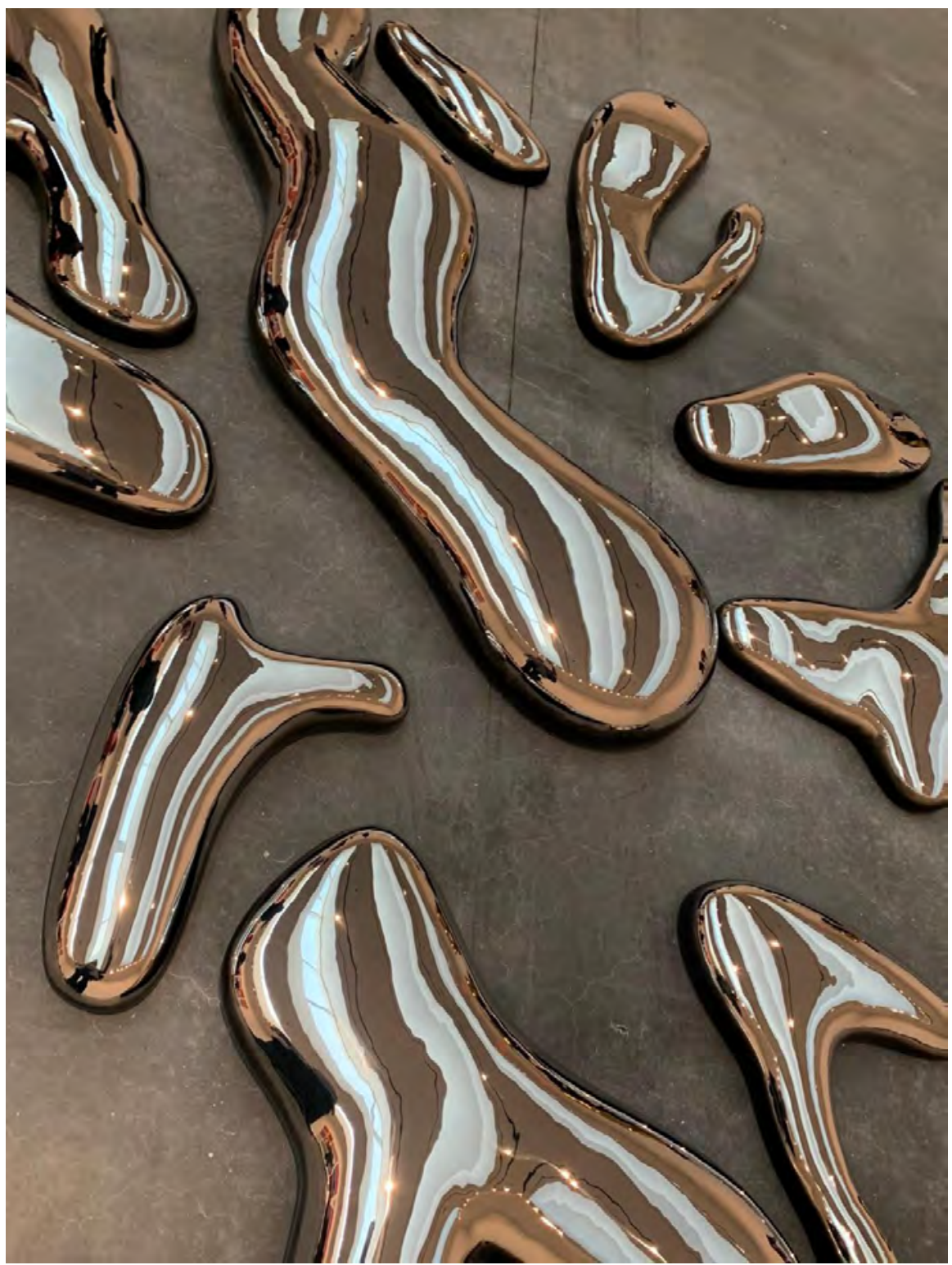

La exhibición continuaba en el segundo piso en donde aislada en un cuarto oscuro se encontraba la pieza de instalación de luz titulada Ladder to Heaven (Escalera al cielo) construida en aluminio y tubos LED colocada en posición 
vertical entre dos espejos circulares, uno en el piso y otro en el techo. La escalera cambiaba de color entre tonos cálidos y fríos y al acercarse y mirar hacia arriba ó abajo se podía ver como la escalera se volvía infinita siguiendo con la temática de su obra. Uno se pregunta si la intención de Kusama al titular su pieza es en realidad unir cielo y tierra continuamente sin fin.

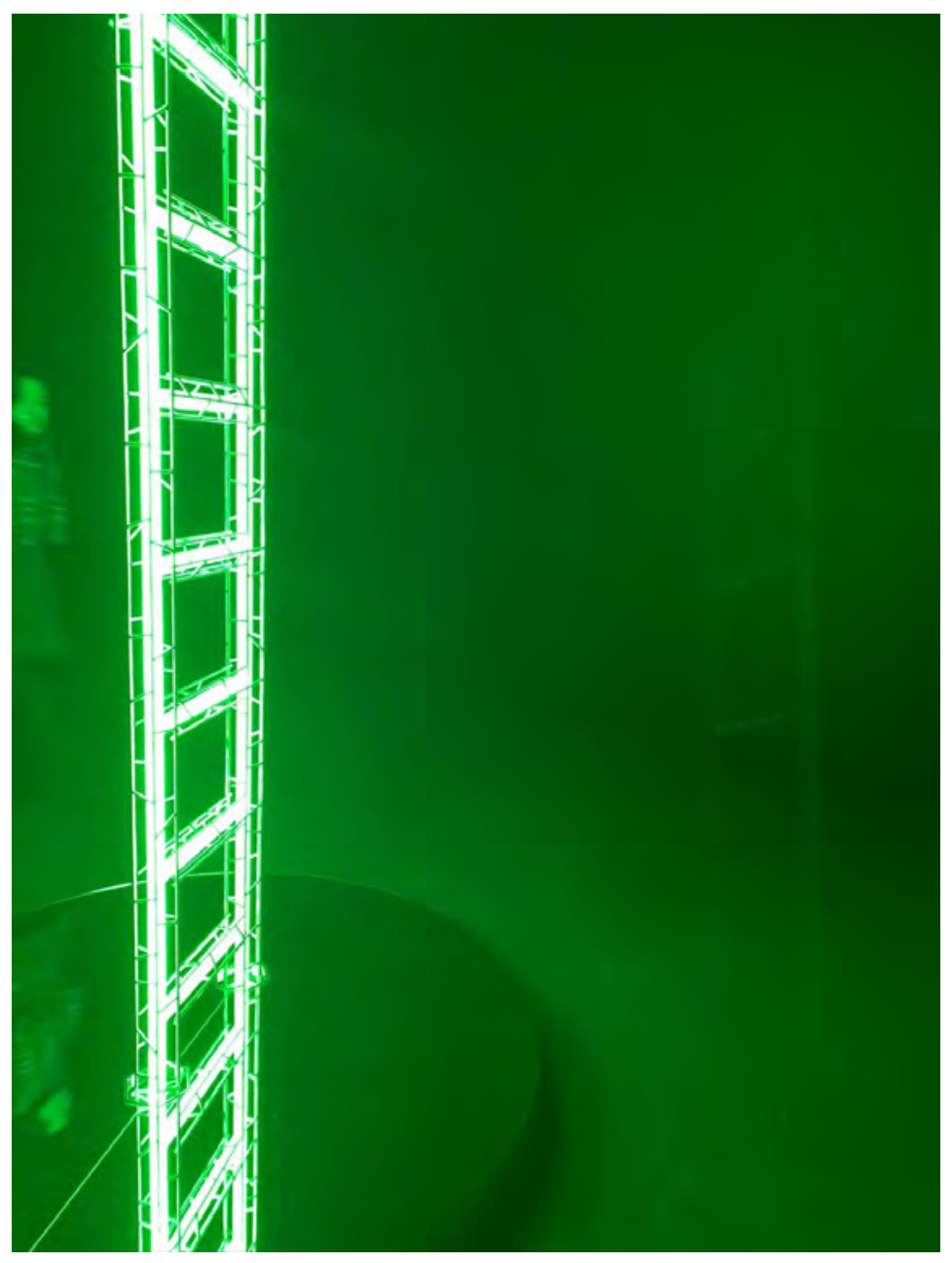

Finalmente, en otra área se exhibía un conjunto de esculturas hechas de tela rellena con espuma y pintadas en su característico estilo de lunares, en donde juega con la repetición de formas y colores - creando estructuras parecidas a plantas - que están colocadas en forma dispersa para poder apreciarlas a corta distancia. 


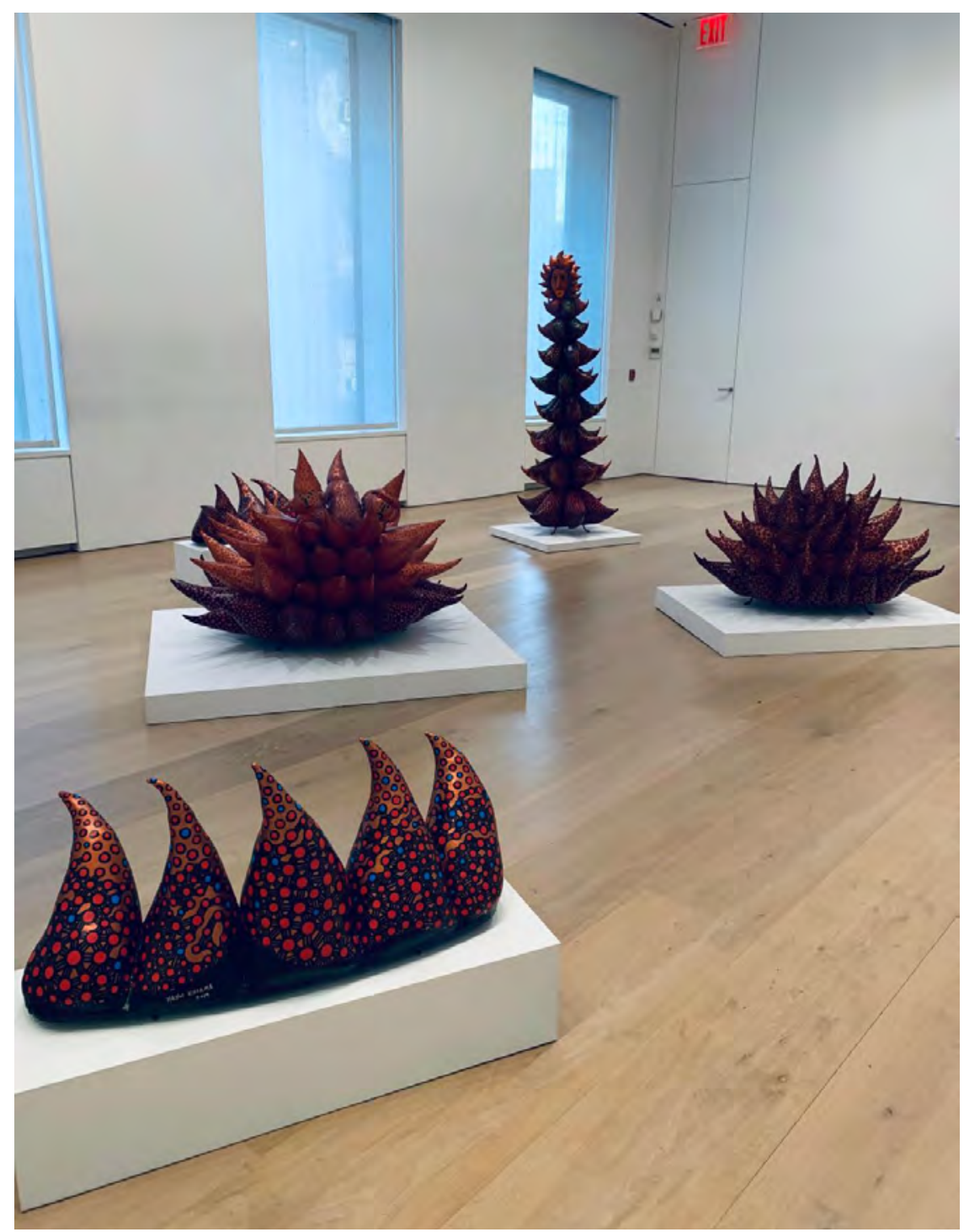

En la pared una serie de trabajos en aluminio fundido en colores y formas diversas cerraba la exhibición, al menos en el recorrido que decidí realizar. Los asistentes tenían la libertad de escoger por dónde empezar si preferían o no hacer la fila de espera de más de una hora para poder entrar a la instalación de cuarto de infinito reflejado, uno de los trabajos más populares de la artista, existiendo alrededor de 19 hasta ahora y una de las tendencias en redes sociales como Instagram. Esta popularidad se debe a que la artista ha logrado recrear en estos espacios la inmensidad de un universo único y nos da la oportunidad de visualizarnos en él. 


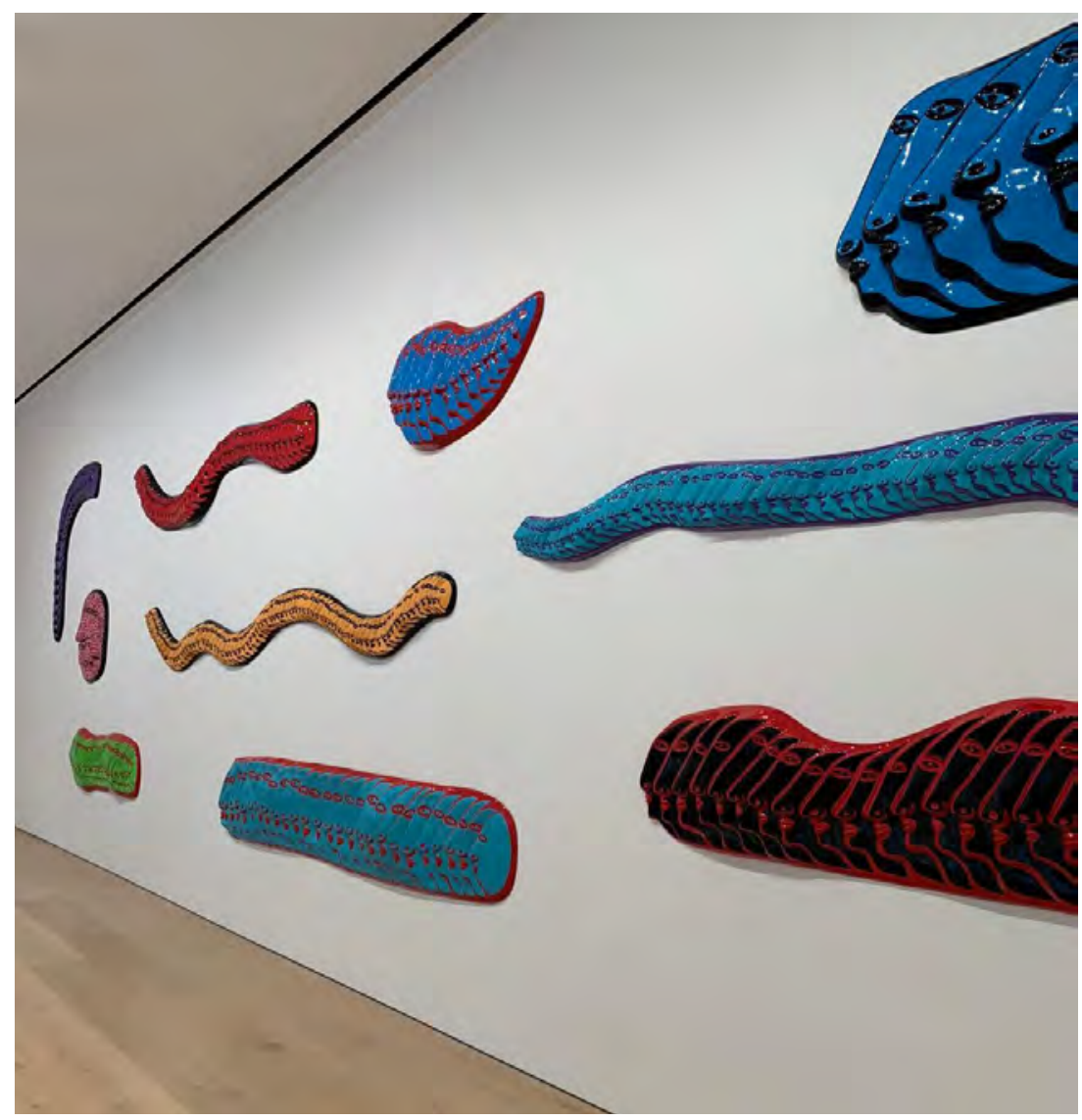

Y es que Yayoi Kusama ha evolucionado a través del tiempo a un arte de estilo inimitable que ha encontrado resonancia en nuestro tiempo ya que su trabajo busca resaltar los sentimientos más puros en el ser humano como el amor y la espiritualidad creando obras que subliman a la naturaleza y el cuál gracias a los medios de comunicación social ha llegado a una audiencia global.

\section{Referencias bibliográficas}

Miro, V. (2019). Yayoi Kusama. Recuperado de https://www.victoria-miro.com/ exhibitions/382/works/96443c0e42e4f0/

The Broad. (2017, octubre 24). Assistant Curator Sarah Loyer discusses Yayoi Kusama's "Off-cut of Infinity Net" painting, a 33-foot work from 1960, and "Phalli's Field," her first Infinity Mirror Room, created in 1965. [Archivo de video]. Recuperado de https://fb.watch/2SSpEjhzkJ/

Zwirner, D. (2019). Yayoi Kusama [versión de Adobe Acrobat Reader]. Recuperado de https://www.davidzwirner.com/artists/yayoi-kusama/-/ media/A351444E009749C9B77DCC9F6481A919.ashx 


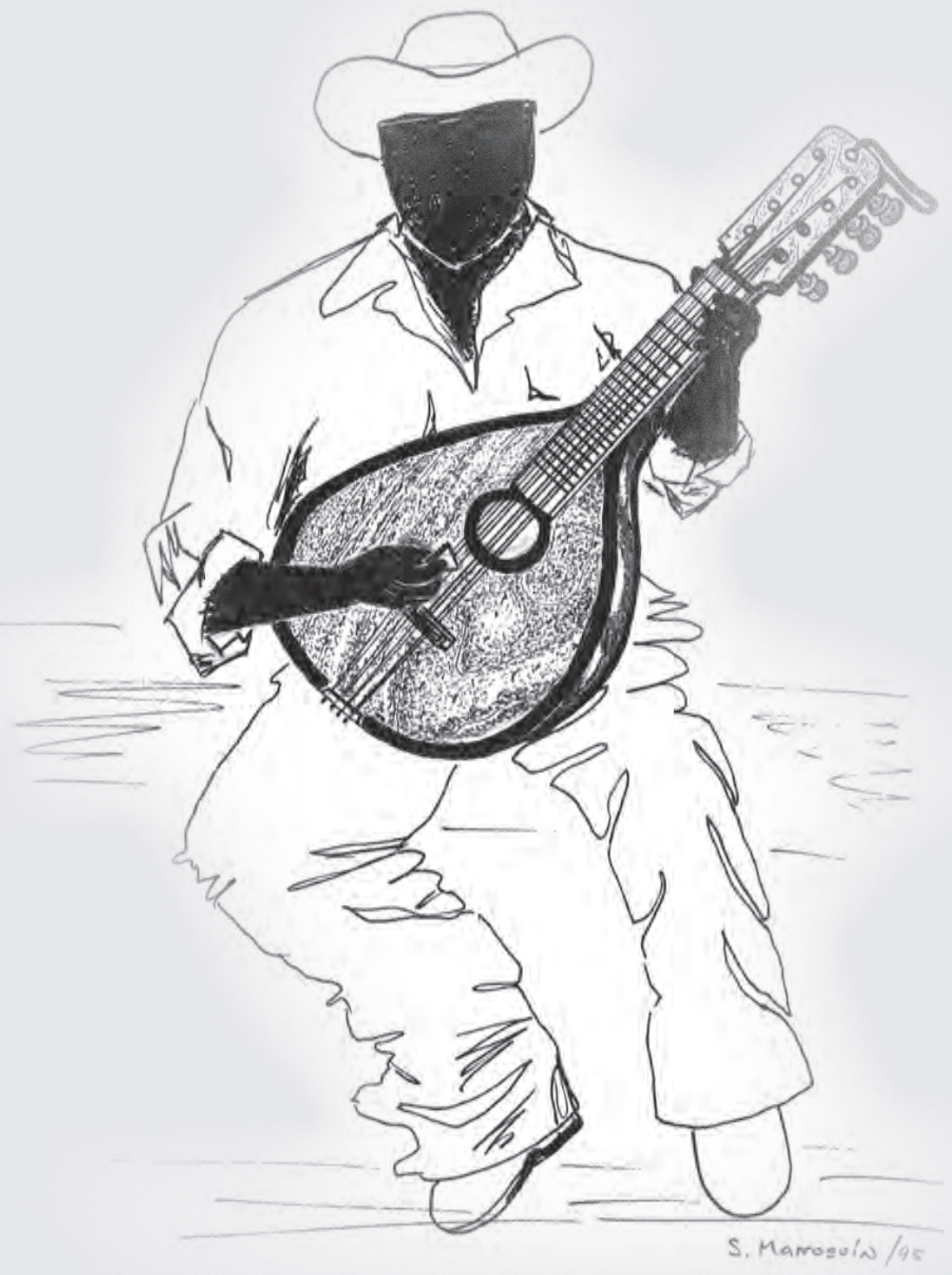

Mandolina

Salvador Marroquín 